\title{
Immune Responses in Neurodegenerative Diseases
}

\author{
Shrestha ${ }^{1},{ }^{1}$ Shakya Shrestha S, ${ }^{1}$ Millington O, ${ }^{2}$ Brewer J, ${ }^{3}$ Bushell ${ }^{2}$
}

${ }^{1}$ Department of Pharmacology

Dhulikhel Hospital - Kathmandu University Hospital

Kathmandu University School of Medical Sciences

Dhulikhel, Nepal

${ }^{2}$ Strathclyde Institute of Pharmacy and Biomedical Sciences, University of Strathclyde Glasgow, UK

${ }^{3}$ Institute of Infection, Immunity and Inflammation College of Medical, Veterinary and Life Sciences University of Glasgow, UK

\section{Corresponding Author}

Rajeev Shrestha

Department of Pharmacology

Dhulikhel Hospital - Kathmandu University Hospital

Kathmandu University School of Medical Sciences

Dhulikhel, Nepal

Email:rmaleku@hotmail.com

\section{Citation}

Shrestha R, Shakya Shrestha S, Millington O, Brewer J, Bushell T. Immune Responses in Neurodegenerative Diseases. Kathmandu Univ Med J 2014;45(1):67-76.

\begin{abstract}
Neurodegenerative disease is a progressive loss of neurons from central nervous system and has a huge impact on health care system. Various causes have been proposed of which inflammation has been suggested to be a probable key factor in the most of such conditions. The involvement of immune cells including lymphocytes in such diseased condition of the CNS supports this notion. The effective therapy for these diseases has been sought for more than a half century but still lacking such therapy. On such basis this review article has mainly focussed on evidence of the involvement of immune cells in various neurodegenerative diseases including Alzheimer's disease, Parkinson's diseases and Multiple sclerosis and suggests a possible therapy of such diseased conditions of the CNS by the modulation of immune system.
\end{abstract}

\section{KEYWORDS}

Alzheimer's disease, lymphocytes, multiple sclerosis, neurodegenerative diseases, parkinson's disease, stroke, $t$ cells

\section{INTRODUCTION}

The global burden of neurodegenerative diseases is huge and accounts for at least $15 \%$ of the global burden of diseases. ${ }^{1}$ Although cases of neurodegenerative disease are increasing every year, reliable therapeutics are still being sought and a complete understanding of the underlying biology is lacking.

Neurodegenerative disease is a progressive loss of neurons from the CNS, associated with a deficit in the function of the affected region. Cell death during neurodegeneration can be either via apoptosis or necrosis or both., ${ }^{2,3}$ Various conditions have been suggested for the neurodegeneration of the CNS including ageing, inflammation, stress and trauma and genetic predisposition. ${ }^{4-11}$ Recent studies have shown a strong link between inflammation and neurodegeneration but the exact role for inflammation in neurodegeneration is still elusive..$^{2,7,12}$ It is not clear whether inflammation causes neuronal death in neurodegenerative diseases or whether the inflammatory infiltrate is simply a manifestation of the disease process. However, several possibilities have been proposed for the link between inflammation and neurodegeneration: 1) inflammation induces neurodegeneration, 2) neurodegeneration causes inflammation, 3) other factors cause either inflammation or neurodegeneration or both, 4) inflammation and neurodegeneration occur as a cycle which amplifies each others response and 5) inflammation can be neuroprotective in neurodegeneration. ${ }^{13}$ The key features of CNS inflammation are glial cell activation, local production of inflammatory mediators, expression of $\mathrm{MHC}$ and adhesion molecules, release of free-radicals and 
recruitment of immune cells. ${ }^{7}$

During neurodegenerative diseases, either peripheral immune cells, such as $\mathrm{T}$ cells, initiate inflammation in the CNS or CNS resident immune competent cells such as microglia as well as neurons, astrocytes and oligodendrocytes, release inflammatory mediators to recruit more peripheral immune cells including lymphocytes leading to CNS inflammation. ${ }^{7,14-17}$ Most commonly, inflammation starts within subarachnoid space which disseminates to other regions of the brain. ${ }^{16-18}$ During inflammation of the CNS, endothelial cells of the BBB express various selectins and adhesion molecules that increase the migration of lymphocytes from the systemic circulation to the perivascular spaces of the brain. ${ }^{19-21}$ Further, activated lymphocytes also express various receptors including chemokines receptors, integrins and selectins that help to interact with their respective ligands expressed on the surface of endothelial cells during neuroinflammation. ${ }^{22-25}$ Activated lymphocytes and cells of the CNS including microglia, astrocytes, neurons and oligodendrocytes release various pro-inflammatory cytokines such as IL-1, TNF- $\alpha$, IL-23, INF- $\gamma$ and chemokines including various neurotrophic factors which can contribute in the outcome of the CNS inflammation. ${ }^{15,26}$ There are several neurodegenerative diseases including $A D, M S, P D$ and stroke in which lymphocytes are actively involved and believed to be a key player in the initiation of CNS inflammation. Some examples of the most common neurodegenerative diseases are briefly explained in subsequent headings.

Alzheimer's disease

Alzheimer's disease (AD) is a progressive neurodegenerative disease that mostly affects patients in their later stage of life. ${ }^{27}$ Typical symptoms of $A D$ are loss of cognitive functions including emotion, learning and memory processing skills leading to dementia. ${ }^{28,29}$ The pathological impression of $A D$ can be characterised by the deposition of amyloid-beta $(A \beta)$ protein plaques in the brain parenchyma and accumulation of tau proteins within neurons. ${ }^{30}$ These protein plaques are thought to interfere with synaptic transmission and neuron-neuron communication leading to neuronal death. ${ }^{28,31,32}$ Further, high levels of tau proteins within neurons form tangles and block transportation of nutrients or other vital cellular factors throughout the cell which has been suggested to be one of the reasons for cell death in AD. ${ }^{31,33,34}$

In $A D$, amyloid-beta plaques and tau proteins are considered to be crucial in the pathology. Recently, inflammation has been proposed to be one of the key players in AD..$^{35-37}$ The inflammatory responses in AD can be characterised by the up-regulation of cytokines and chemokines along with activation of microglia. ${ }^{35}$ The activated microglia clusters can be seen near amyloid-beta deposition site and these cells also express high levels of MHC-II, cytokines and chemokines contributing to disease progression. ${ }^{35,38-41}$
However, these microglial cells also involve in clearing of amyloid-beta and this function has been shown to be enhanced in the presence of TGF- $\beta .^{37,42}$ In addition, reactive astrocytes are also clustered at the site of amyloid-beta deposition but high levels of these cells also accumulate at neuritic plaques. ${ }^{43}$ These reactive astrocytes are capable of expressing various cytokines, growth factors, adhesion molecules and prostaglandins. ${ }^{44-48}$ Furthermore, astrocytes are suggested to be involved in inhibition of microglial ability to clear amyloid beta. ${ }^{49}$ The analysis of brain autopsy has also revealed that there is a significant increase in inflammatory markers as well as increase in complement activation and lysis of neurites in AD subjects when compared to non-demented subjects, which strongly suggests that inflammation has a role in $A D .{ }^{50,51}$ With these findings, it can be suggested that this inflammatory responses might contribute in recruiting lymphocytes from the systemic circulation into the brain. Further, T cells have been detected in the brain of AD patients. ${ }^{52}$ Recent studies have also demonstrated the up-regulation of $T$ cells in the CNS of AD when compared to healthy controls. ${ }^{53,54}$ These studies have revealed an increased activity of various subsets of T cells such as Th- 17 and Th- 9 in AD and cytokines including IL-9, IL-21 and IL-23 released from these T cells are also increased in $A D$ which have been suggested to be one of the factors in AD-associated neuroinflammation. ${ }^{53}$ The beneficial effect of $T$ cells has been demonstrated in $A D$ however, these T cells lose their protective effect in severe condition of $A D .{ }^{54}$ With these findings, it is evident that lymphocytes are present in the brain of $A D$ and may have an important role in AD. However, the role of lymphocytes in $A D$ is still poorly understood and a better understanding of this phenomenon could help in search of novel drug targets.

\section{Multiple sclerosis}

Multiple sclerosis (MS) is a chronic neuroinflammatory disease of the CNS characterised by demyelination, axonal damage and autoimmunity affecting people mostly between of 20 and $40 .{ }^{55-58}$ It affects both white and gray matter of the CNS and scattered focal demyelinated lesions can be seen throughout the white matter of the CNS. ${ }^{55,59}$ There is an episodic exacerbation followed by remission during the course of the disease and this relapsing-remitting course is suggested to be immune-mediated which is characterised by activation of microglia and infiltration of peripheral immune cells into the CNS. ${ }^{57,60}$ This disease process leads to secondary MS having marked degeneration of neurons and axons along with massive cortical demyelination. ${ }^{55,57,61,62}$

Genetic predisposition, environmental factors and viral/ microbial infections have been proposed as risk factors in MS but how these factors contribute to the aetiology of the disease is still under investigation. ${ }^{63-67}$ However, it is now well accepted that MS is a T cell mediated autoimmune disease. ${ }^{13,56,60,68}$ The concept of MS as an autoimmune disease arises due to its similarities in clinical symptoms with $E A E$, an animal model for this disease. ${ }^{69} E A E$ is 
induced in the animal by immunising with myelin-derived protein such as myelin-basic protein, proteolipid protein or myelin oligodendrocyte glycoprotein and the disease is mainly initiated by myelin-specific autoreactive T cells. Autoreactive T cells have been identified in MS patients, as well as being present in healthy people. ${ }^{70}$ However, these T cells are more activated and have a memory phenotype in MS when compared to healthy subjects. ${ }^{62,71}$ These activated $T$ cells express various chemokines, cytokines and adhesion molecules which help them to interact with the BBB and migrate into the CNS to initiate immune response against myelin-derived proteins. Although, CD4+ T cells are thought to be initiators of EAE, analysis of MS lesioned brain tissues has shown predominance of $\mathrm{CD} 8+\mathrm{T}$ cells. ${ }^{72} \mathrm{CD} 4+\mathrm{T}$ cells can be either neuroprotective or pathogenic depending on the types of cytokines or neurotrophic factors they release. ${ }^{13}$ As described above, different subsets of CD4+ T cells release their functional cytokines: Th1 cells release inflammatory mediators including IFN- $\gamma$ and TNF- $\alpha$ whereas Th- 2 cells release anti-inflammatory mediators like IL-4. ${ }^{73}$ IFN- $\gamma$ and TNF- $\alpha$ have shown a contrast in response in EAE and MS since IFN- $\gamma$ exhibits neuroprotection in rodent models of EAE whereas it exacerbates the disease in MS. ${ }^{13,74}$ Similarly, blocking TNF- $\alpha$ function in EAE is neuroprotective but not in $M S .{ }^{75}$ This finding therefore suggests that caution should be taken while comparing data from the EAE model with MS. The major concept of immunopathogenesis of MS has been connected to the balance between Th- 1 and Th- 2 functions. However, there are other subsets of CD4+ T cells such as Th-17 and Treg cells along with CD8+ T cells cannot be excluded. Th-17 cells have been found to express more activation markers, co-stimulatory and adhesion molecules than Th- 1 cells suggesting they are more pathogenic. Further, the pathogenic T cells suppressor capacity of Treg cells is found to be attenuated in MS. ${ }^{76,77}$ Moreover, CD8+ $T$ cells have been directly linked to the demyelination of axons in MS and are pathogenic in the immune-mediated demyelination of axons. ${ }^{75,78}$ Nevertheless, there is no exact mechanism how these cells are contributing to the disease process or neuroprotection in MS and further research is required to understand MS pathology.

Parkinson's disease

Parkinson's disease (PD) is an age-related chronic neurodegenerative disease clinically characterised by tremor, rigidity, bradykinesia, postural instability, dementia and autonomic dysfunction while pathologically by loss of dopaminergic neurons in the substantia niagra and the presence of Lewy's bodies which are aggregated proteins such as $\alpha$-synuclein. ${ }^{32,79-81}$ The accumulation and misfolding of $\alpha$-synuclein induce toxicity leading to the loss of dopaminergic neurons. ${ }^{82}$ This results in a reduction of dopamine production causing gait and movement impairment because dopamine is required for a normal motor function of the brain. ${ }^{79}$ Further, proteosomal and lysosomal system dysfunction and reduction in mitochondrial activity due to genetic mutations are also proposed to be causative factors in neuronal death during PD. ${ }^{82}$ Various risk factors including environmental genetics and age have been related to pathogenesis of PD. $4,80,83,85$ Furthermore, there are several studies suggesting the relation between inflammation and pathology of PD. 86,88 However, it is still not clear whether inflammation observed in PD can be considered as classical inflammation or not. ${ }^{86}$ Therefore, the term 'neuroinflammation' has been coined in the pathology of PD. The upregulation of $\mathrm{MHC}$ expression is one of the first signs of neuroinflammation in PD with an increase in MHC-II expressed microglia in the substantia niagra. ${ }^{86,89}$ Similar upregulation of $\mathrm{MHC}$ molecules has been reported in 1-methyl-4-phenyl-1,2,3,6tetrahydropuridine (MPTP)-intoxicated animals, an animal model for PD and is also associated with the infiltration of lymphocytes in substantia niagra. ${ }^{90}$ The other hallmarks for neuroinflammation are the presence of reactive astrocytes, activated microglia, increase in cytokines, chemokines, prostaglandins, and reactive oxygen and nitrogen species. ${ }^{91}$ Microglial activation has been related to accumulation of $\alpha$-synuclein protein and proteosomal and lysosomal system dysfunction and these activated microglial cells have been reported to induce cell death in dopaminergic neurons. ${ }^{88,92}$ Furthermore, neurons over-expressing $\alpha$-synuclein protein have demonstrated early activation of microglia and release of various inflammatory mediators such as IL-1, IL- 6 and TNF- $\alpha$ and inflammation-related enzymes including cylcoxygenase-2 (COX-2) and inducible nitric oxide synthase. ${ }^{93-96}$ In addition, pro-inflammatory factors including IL-1, TNF- $\alpha$, reactive oxygen/nitric oxide species and prostaglandins released from the activated microglia induced by $\alpha$-synuclein protein can enhance oxidative stress and trigger cell-death pathways. ${ }^{97-99}$ These locally released inflammatory mediators also induce expression of cellular adhesion molecules in the endothelium of the BBB which help in the subsequent recruitment of immune cells from the periphery into the inflammatory site. ${ }^{100}$ Several studies have shown the infiltration of lymphocytes into the CNS in PD. ${ }^{101-104}$ These immune cells including leukocytes, macrophages, $B$ cells and $T$ cells, predominantly, CD4+ T cells and $C D 8+T$ cells are recruited in the substantia niagra region of the brain in $\mathrm{PD}$ with $\mathrm{CD} 4+\mathrm{T}$ cells being suggested to be cytotoxic in PD. ${ }^{102,102}$ However, the other subsets of $T$ cells such as Treg cells are also present in the substantia niagra of PD and have the ability to suppress the cytotoxicity of effector T cells like Th-17 cells. ${ }^{105,107}$ These Treg cells can also induce apoptosis in activated microglia and reduce $\alpha$-synuclein protein induced neurotoxicity. ${ }^{106,107}$ Although various studies have argued on either the pathogenic or the neuroprotective role of lymphocytes, the precise role of lymphocytes in PD is still elusive. Hence, it is evident that lymphocytes play a key role in PD but, their role in relation to PD pathology still needs to be explored extensively.

Stroke

The World Health Organisation (WHO) has defined stroke as the clinical syndrome of rapid onset of focal or global 
cerebral deficit, lasting more than 24 hours or leading to death with no apparent cause other than a vascular one. ${ }^{108}$ Various risk factors including smoking, diabetes, hypertension, atrial fibrillation and transient ischaemic attack have been identified for stroke which can lead to either haemorrhagic or ischaemic stroke. ${ }^{109}$ Haemorrhagic stroke is due to hypertensive arteriosclerosis and amyloid angiopathy whereas, the major cause of focal or ischaemic stroke is due to the occlusion of major arteries of the brain and accounts for $80 \%$ of all strokes. ${ }^{110,111}$ The occlusion in the artery within the CNS leads to a reduction of blood flow leading to insufficient oxygen and glucose supply to the brain. Usually, this occlusion develops due to thrombosis in situ (such as atherothrombosis of large cervical or intracranial arteries), or embolism from heart or blockage of small penetrating arteries due to arteritis or haematological disorders. ${ }^{112}$ In stroke, a series of neurochemical processes occurs which is termed an ischaemic cascade. During ischaemic cascades, various events take place including cellular metabolic failure due to hypoperfusion, oxidative stress, excitoxicity, damage of BBB, microvascular injury, activation of haemostatic system and inflammation. ${ }^{113}$ These series of events result in non-selective cell death in the CNS including neurons, astrocytes, microglia, oligodendrocytes and endothelial cells. ${ }^{114}$

Apart from various risk factors including smoking, diabetes and hypertension, there are several studies showing a relation between inflammatory status and the risk of stroke which also has an effect on the outcome of stroke. ${ }^{115-118}$ The inflammatory response arises from the series of ischaemic cascades. The hypoperfusion occurs during this cascade causes a failure of energy-dependent ion pump leading to activation of calcium channels and release of excitatory neurotransmitters including glutamate into extracellular spaces. ${ }^{119-121}$ The release of excess excitatory neurotransmitters causes neuronal death which is termed as excitotoxicity. ${ }^{113,122-124}$ On the other hand, a disturbance in the scavenging of free reactive radicals causes oxidative stress. ${ }^{113,125,126}$ There is significant evidence illustrating the cytotoxic effects of free reactive oxygen and nitrogen species. ${ }^{127-129}$ This oxidative stress leads to glial cells activation followed by release of various inflammatory mediators including cytokines, chemokines and reactive free radicals, as well as expression of MHC I and II and co-stimulatory molecules. ${ }^{118,130,131}$ The level of IL-1 has been reported to be elevated after experimental stroke and has been a target for therapy in stroke in reducing inflammation-related damage. ${ }^{115,132-134}$

Non-specific activation of peripheral $T$ cells has been reported in stroke patients compared with healthy subjects, and the number of Treg cells is increased in stroke patients, similar to that described in animal models. ${ }^{135,136}$ During inflammation, endothelial cells of the $B B B$ express various adhesion molecules including ICAM-1, ICAM-2 and VCAM-2 and selectins that help lymphocytes migration from the periphery to the CNS. ${ }^{137-}$ 140 These lymphocytes express chemokine receptors, $\alpha 4 \beta 1$ integrins and LFA- 1 helping them in capturing, activation and transendothelial migration across the BBB. ${ }^{22,100}$ Several studies have shown infiltration of lymphocytes into the CNS following stroke. ${ }^{141-144}$ Real time in vivo imaging of the mouse brain after experimental stroke revealed a massive number of $\mathrm{T}$ cells infiltration into the stroke brain in comparison to sham. ${ }^{141}$ This recent study has reported two distinct populations of T cells in experimental stroke, the fast migrating $T$ cells and slow migrating $T$ cells but, the definitive role of these two populations in stroke is still under investigation. ${ }^{141}$ Furthermore, various studies have shown the neurotoxic effect in the stroke while at the same time others have argued on their neuroprotective role in the stroke. ${ }^{145-149}$ Taken together, to date there has been little agreement on the precise role of lymphocytes in the stroke and a better understanding of their contribution to stroke is still required.

Role of lymphocytes in neurodegenerative diseases: pathogenic or neuroprotective?

Infiltration of lymphocytes into the CNS during neurodegenerative diseases is well established and the molecular mechanisms underlying their recruitment into the CNS has also been well documented. ${ }^{13,22,53,56 \text {, }}$ $102,104,141,100,150$ However, the controversy of scientific evidence for the role of lymphocytes during neurodegeneration has raged unabated for more than a half century.

It is now evident that after infiltration into the CNS and recognition of cognate antigen/MHC, peripherally activated lymphocytes (see section 1.3.1) can initiate inflammatory response in the CNS which can be either neuroprotective or neurotoxic. 22,151 The pathogenic role of T cells has been demonstrated in neurodegenerative diseases causing neuronal death. ${ }^{101,103,152,153}$ The extent of the CNS injury during neurodegeneration has been correlated with the increase in T cells infiltration into the CNS suggesting the greater the infiltration, the greater the neuronal injury. ${ }^{154}$ It has been suggested that these infiltrating $T$ cells can also mediate cell death and demyelination in neurodegenerative diseases, affecting other effector cells including mircoglia and/or macrophages. ${ }^{155}$ The adoptive transfer of $\mathrm{T}$ cells from spinal cord injury model mice and EAE-induced mice to healthy recipients develops paralytic disease which further supports the pathogenic role of T cells. ${ }^{155}$ It has been shown that during neurodegeneration and brain injury, both $T$ cells and $B$ cells are activated which is referred to as auto-reactive T cells or B cells. ${ }^{156,157}$ The number of auto-reactive T cells is increased in neurodegeneration and CNS trauma and they predominantly release IFN- $\gamma$ and TNF- $\alpha .^{154,156,158}$ Moreover, these cytokines released by these auto-reactive cells can exacerbate ischaemia and excitotoxicity in the brain during neurodegeneration. ${ }^{159}$ Studies have also demonstrated that TNF- $\alpha$ induces cell death via apoptotic pathways and its concentration was also found to be elevated during neurodegenerative disease including PD. ${ }^{160,161}$ In addition, 
activated CD4+ T cells express Fas-ligand (FasL), which has been reported to induce cell death via apoptosis in neurodegenerative diseases including EAE. ${ }^{162,163}$ These Fas and FasL are type I and II transmembrane receptors belonging to TNF/nerve growth factor and TNF families' protein respectively. ${ }^{164}$ The infiltrating CD4+ T cells in PD induce apoptosis of dopaminergic neurons via FasL-Fas interaction, mediating FasL-mediated activation of microglia and neurodegeneration. ${ }^{101}$ The up-regulation of Fas and their ligands have been demonstrated in the CNS during neurodegenerative disease such as EAE leading to the apoptotic cell death. ${ }^{165}$ In addition, CD8+ T cells or cytotoxic $\mathrm{T}$ lymphocytes (CTL) are proposed to be involved in direct killing of neurons in a MHC-I dependent manner. ${ }^{166,167}$ The induction of MHC-I expression in neurons via IFN- $\gamma$ has been documented and it has also been reported that the cytotoxicity of CTL in these neurons is mediated via either FasL-mediated neuronal apoptosis or perforin-dependent lysis of neurons. ${ }^{167-169}$ Moreover, both CD4+ T cells and CD8+ $T$ cells have been reported to be equally neurotoxic and mediated via direct cell contact mechanism involving FasL, LFA-1 and CD40. ${ }^{170}$

Despite the proposed role of T cells in neurodegneration, there is growing evidence for a beneficial or neuroprotective role of lymphocytes in neurodegenerative diseases. ${ }^{171-175}$ Adoptive transfer of auto-reactive $T$ cells from EAEinduced mice to healthy recipient induces pathology. ${ }^{155}$ However, when these cells are transferred to the mice with partial optic nerve crush, a model for secondary neurodegeneration, they were found to be beneficial. ${ }^{173}$ Nerve cells from the mice which received auto-reactive $T$ cells were found to survive well and were resistant to secondary neurodegeneration. Further analysis also revealed that only $\mathrm{T}$ cells specific to MBP were able to protect retinal ganglion cells from secondary damage and not $T$ cells specific for non-self antigens, such as ovalbumin and heat-shock proteins. They further have suggested that only CNS-specific T cells are activated at the injured site to exert the neuroprotective response while $T$ cells specific to non-self antigens fail to activate because of inadequate antigen recognition. ${ }^{173}$ In addition, after experimental axotomy of facial nerves, facial motor neurons are found to be severely impaired in severe combined immunodeficient (SCID) mice which lack T cells and B cells. ${ }^{176,177}$ but are restored up to wild-type controls after adoptive transfer of wild-type splenocytes containing $T$ and B cells. Similarly, intraperitoneally injection of auto-reactive MBP-specific $T$ cells in rat with an experimentally crushed spinal cord show early recovery with greater locomotor function as compared to controls. ${ }^{178,179}$ Moreover, an in vitro study in murine entorhinal-hippocampal brain slices shows downregulation of the Th-1 cells induced inflammatory marker, ICAM-1 in microglia while another study demonstrated the neuroprotective role of both Th- 1 and Th-2 cells. ${ }^{175,180}$

Itisnowwellunderstood that uponactivation bytheircognate antigen/MHC-II, CD4+ T cells in the presence of appropriate mediators also differentiate into Treg cells and several studies have demonstrated the immunosuppressive role of Treg during neuroinflammation. ${ }^{105,107,136,181-185}$ Treg cells have an immunomodulatory role in human immunodeficiency virus (HIV)-1-induced neurodegeneration leading to neuroprotection by suppressing microglial activation and secreting neurotrophic factors. ${ }^{185}$ Treg cells are able to salvage neurons by suppressing the inflammatory response mediated by Th-17 cells. ${ }^{105}$ It has also been demonstrated that interaction between neurons and pathogenic T cells in EAE-induced mice cause conversion of pathogenic T cells into Treg cells which are able to suppress neurodegeneration induced by pathogenic T cells. ${ }^{184}$ The conversion of pathogenic T cells into neuroprotective Treg cells has been suggested to be induced by the interaction of CD4+ T cells and neurons via B7-CD28 and TGF- $\beta$ receptor signalling pathways. ${ }^{184}$ In addition, IL-10 producing CD4+ T cells are found to be neuroprotective in stroke and it has been demonstrated that IL-10 and TGF- $\beta$ can modulate immune processes by inhibiting Th-1-induced inflammatory responses as well as general inflammation leading to neuroprotection. ${ }^{147,186,187}$ These auto-reactive T cells are able to produce various neurotrophic factors including brain-derived neurotrophic factors (BDNF), neurotrophin-3 (NT-3) and glial-cell derived neurotrophic factors which can rescue neurons from neurodegeneration. ${ }^{172,188}$ It has also been suggested that $T$ cells can instruct microglia to remove neurotoxic extracellular glutamate resulting in neuroprotection. ${ }^{189}$

Investigating the role of $B$ cells in $E A E$-induced mice reveals that $B$ cell deficient mice are more susceptible to EAE induction developing severe pathology with delayed recovery and early demyelination in compare to their controls. ${ }^{190,191}$ Likewise, a decrease or absence of B cells in EAE-induced mice correlates with an increase in severity of disease as well as influx of more pathogenic T cells into the CNS. ${ }^{192,193}$ In addition, IL-10 secreted by B cells is reported to be neuroprotective in EAE-induced mice since $E A E$ induced mice having IL-10 deficit B cells failed to recover and manifested persistent inflammatory responses. ${ }^{194}$ Hence, IL-10 specific B cells are suggested to be playing a crucial role in the recovery and progression of the disease. ${ }^{193}$ It has also been demonstrated that $\mathrm{B}$ cells are able to limit the spreading and severity of disease in EAEinduced mice. ${ }^{195}$ Furthermore, there is evidence showing that $B$ cells are able to release various neurotrophic factors including BDNF, NT-3 and neurotrophic growth factor which have a possible role in contributing to neuroprotection in neurodegenerative diseases. ${ }^{196-198}$ In addition, recent paper has shown the neuroprotective role of lymphocytes during neurodegeneration either induced by excitotoxicity or glucose-oxygen deprivation which is mediated through the astrocytic activation and modulation of mitogen activated protein (MAP) kinases. ${ }^{199} \mathrm{IL}-6$ has been proposed to be one of the potential factors in lymphocyte mediated neuroprotection. ${ }^{199}$ 
Thus, it is clear that the notion of the brain as an absolute immune-privileged site is no longer appropriate, rather it can be considered as a site of active immune-surveillance. Recent evidence suggests the existence of bidirectional communication between these two systems and can influence each other via various mediators in both healthy and diseased condition of the CNS. In addition to this bidirectional communication, several studies have reported that lymphocytes are regularly patrolling the normal CNS in low number which can be increased upon recognition of cognate antigen and initiation of inflammation at the site. Whilst many attempts have been made to describe

\section{REFERENCES}

1. Cruz, M., R. Jenkins, and D. Silberberg, The Burden of Brain Disorders. Science. 2006; 312(5770): 53 .

2. DeLegge, M.H. and A. Smoke, Neurodegeneration and inflammation. Nutr Clin Pract. 2008; 23(1): 35-41.

3. Martin, L.J. Neurodegeneration in excitotoxicity, global cerebral ischemia, and target deprivation: A perspective on the contributions of apoptosis and necrosis. Brain Res Bull. 1998; 46(4): 281-309.

4. Collier, T.J., N.M. Kanaan, J.H. Kordower. Ageing as a primary risk factor for Parkinson's disease: evidence from studies of non-human primates. Nat Rev Neurosci. 2011; 12(6): 359-366.

5. Tollervey, J.R. Analysis of alternative splicing associated with aging and neurodegeneration in the human brain. Genome Research, 2011.

6. Amor, S. Inflammation in neurodegenerative diseases. Immunology. 2010; 129(2): 154-169.

7. Lucas, S.M., N.J. Rothwell, R.M. Gibson, The role of inflammation in CNS injury and disease. Br J Pharmacol. 2006; 147 Suppl 1: S232-40.

8. Bramlett, H.M. and W.D. Dietrich. Pathophysiology of cerebral ischemia and brain trauma: similarities and differences. J Cereb Blood Flow Metab. 2004; 24(2): 133-50.

9. Sugama, S. Stress-induced microglial activation may facilitate the progression of neurodegenerative disorders. Medical Hypotheses. 2009; 73(6): 1031-1034.

10. Butterfield, R.J. Identification of genetic loci controlling the characteristics and severity of brain and spinal cord lesions in experimental allergic encephalomyelitis. Am J Pathol. 2000; 157(2): 637-45.

11. Elbaz, A., C. Dufouil, and A. Alpérovitch. Interaction between genes and environment in neurodegenerative diseases. Comptes Rendus Biologies. 2007; 330(4): 318-328.

12. Campbell, A. Inflammation, neurodegenerative diseases, and environmental exposures. Ann N Y Acad Sci. 2004; 1035: 117-32.

13. Peterson, L.K. and R.S. Fujinami. Inflammation, demyelination, neurodegeneration and neuroprotection in the pathogenesis of multiple sclerosis. J Neuroimmunol. 2007; 184(1-2): 37-44.

14. Hickey, W.F. and H. Kimura. Perivascular microglial cells of the CNS are bone marrow-derived and present antigen in vivo. Science. 1988; 239(4837): 290-2.

15. Neumann, H. Control of glial immune function by neurons. Glia. 2001; 36(2): 191-9.

16. Kivisäkk, P. Localizing central nervous system immune surveillance: Meningeal antigen-presenting cells activate $T$ cells during experimental autoimmune encephalomyelitis. Annals of Neurology. 2009; 65(4): 457-469. either the pathogenic or the neuroprotective role of lymphocytes, there has been little discussion about precise function(s) and mechanism(s) of these responses during neurodegeneration. Therefore, by defining the role and underlying mechanism(s) of lymphocytes and other related immune cells in neurodegenerative diseases, we certainly can set a milestone for the better understanding of the disease pathology and its therapy. Moreover, on this basis, the possibility of immune-based therapy of neurodegenerative diseases cannot be overlooked, though proper pros and cons should be determined beforehand.

17. Block, M.L. and J.-S. Hong. Microglia and inflammation-mediated neurodegeneration: Multiple triggers with a common mechanism. Progress in Neurobiology. 2005; 76(2): 77-98.

18. Lassmann, H. and H.M. Wisniewski. Chronic relapsing EAE time course of neurological symptoms and pathology. Acta Neuropathologica. 1978; 43(1): 35-42.

19. Engelhardt, B. and H. Wolburg. Mini-review: Transendothelial migration of leukocytes: through the front door or around the side of the house? Eur J Immunol. 2004; 34(11): 2955-63.

20. Kivisäkk, P. T-cells in the cerebrospinal fluid express a similar repertoire of inflammatory chemokine receptors in the absence or presence of CNS inflammation: implications for CNS trafficking. Clinical \& Experimental Immunology. 2002; 129(3): 510-518.

21. Reboldi, A. C-C chemokine receptor 6-regulated entry of $\mathrm{TH}-17$ cells into the CNS through the choroid plexus is required for the initiation of EAE. Nat Immunol. 2009; 10(5): 514-523.

22. Engelhardt, B. T cell migration into the central nervous system during health and disease: Different molecular keys allow access to different central nervous system compartments. Clinical and Experimental Neuroimmunology. 2010; 1(2): 79-93.

23. Goverman, J. Autoimmune $\mathrm{T}$ cell responses in the central nervous system. Nat Rev Immunol. 2009; 9(6): 393-407.

24. Piccio, L. Molecular Mechanisms Involved in Lymphocyte Recruitment in Inflamed Brain Microvessels: Critical Roles for P-Selectin Glycoprotein Ligand-1 and Heterotrimeric Gi-Linked Receptors. The Journal of Immunology. 2002; 168(4): 1940-1949.

25. Ransohoff, R.M., P. Kivisakk, and G. Kidd. Three or more routes for leukocyte migration into the central nervous system. Nat Rev Immunol. 2003; 3(7): 569-81.

26. Kerschensteiner, M., E. Meinl, and R. Hohlfeld. Neuro-immune crosstalk in CNS diseases. Neuroscience. 2009; 158(3): 1122-32.

27. Isik, A.T. Late onset Alzheimer's disease in older people. Clin Interv Aging. 2010; 5: 307-11.

28. Mattson, M.P. Pathways towards and away from Alzheimer's disease. Nature. 2004; 430(7000): 631-639.

29. Jalbert, J.J., L.A. Daiello, and K.L. Lapane. Dementia of the Alzheimer Type. Epidemiologic Reviews. 2008; 30(1): 15-34.

30. Krause, D.L. and N. Muller. Neuroinflammation, microglia and implications for anti-inflammatory treatment in Alzheimer's disease. Int J Alzheimers Dis. 2010.

31. Alzheimer's-Association, 2011 Alzheimer's disease facts and figures. Alzheimer's and Dementia. 2011; 7(2): 208-244.

32. Ang, E.T. Neurodegenerative diseases: exercising toward neurogenesis and neuroregeneration. Front Aging Neurosci. 2010: 2. 
33. Ballatore, C., V.M.Y. Lee, and J.Q. Trojanowski. Tau-mediated neurodegeneration in Alzheimer's disease and related disorders. Nat Rev Neurosci. 2007; 8(9): 663-672.

34. Iqbal, K. Tau in Alzheimer disease and related tauopathies. Curr Alzheimer Res. 2010; 7(8): 656-64.

35. Akiyama, H. Inflammation and Alzheimer's disease. Neurobiol Aging. 2000; 21(3): 383-421.

36. Tahara, K. Role of toll-like receptor signalling in $A \hat{l}^{2}$ uptake and clearance. Brain. 2006; 129(11): 3006-3019.

37. Wyss-Coray, T. Inflammation in Alzheimer disease: driving force, bystander or beneficial response? Nat Med. 2006; 12(9): 1005-1015.

38. Griffin, W.S.T. Glial-Neuronal Interactions in Alzheimer's Disease: The Potential Role of a 'Cytokine Cycle' in Disease Progression. Brain Pathology. 1998; 8(1): 65-72.

39. Streit, W.J., S.A. Walter, and N.A. Pennell. Reactive microgliosis. Prog Neurobiol. 1999; 57(6): 563-81.

40. Banati, R.B. Cytotoxicity of microglia. Glia. 1993; 7(1): 111-118.

41. Dickson, D.W. Microglia and cytokines in neurological disease, with special reference to AIDS and Alzheimer's disease. Glia. 1993; 7(1): 75-83.

42. Wyss-Coray, T. TGF-[beta]1 promotes microglial amyloid-[beta] clearance and reduces plaque burden in transgenic mice. Nat Med. 2001; 7(5): 612-618.

43. Mrak, R.E., J.G. Sheng, and W.S. Griffin. Correlation of astrocytic S100 beta expression with dystrophic neurites in amyloid plaques of Alzheimer's disease. J Neuropathol Exp Neurol. 1996; 55(3): 273-9.

44. Del Bo, R. Reciprocal control of inflammatory cytokines, IL-1 and IL6 , and [beta]-amyloid production in cultures. Neuroscience Letters. 1995; 188(1): 70-74.

45. Hampel, H. Pattern of interleukin-6 receptor complex immunoreactivity between cortical regions of rapid autopsy normal and Alzheimerâ $€^{\mathrm{TM}} \mathrm{S}$ disease brain. European Archives of Psychiatry and Clinical Neuroscience. 2005; 255(4): 269-278.

46. Flanders, K.C., R.F. Ren, and C.F. Lippa. TRANSFORMING GROWTH FACTOR-[beta]S IN NEURODEGENERATIVE DISEASE. Progress in Neurobiology. 1998; 54(1): 71-85.

47. Akiyama, H. Expression of intercellular adhesion molecule (ICAM)1 by a subset of astrocytes in Alzheimer disease and some other degenerative neurological disorders. Acta Neuropathologica. 1993; 85(6): 628-634

48. Hirst, W.D. Expression of COX-2 by Normal and Reactive Astrocytes in the Adult Rat Central Nervous System. Molecular and Cellular Neuroscience. 1999; 13(1): 57-68.

49. DeWitt, D.A. Astrocytes Regulate Microglial Phagocytosis of Senile Plaque Cores of Alzheimer's Disease. Experimental Neurology. 1998; 149(2): 329-340.

50. Lue, L.F., et al. Inflammation, A beta deposition, and neurofibrillary tangle formation as correlates of Alzheimer's disease neurodegeneration. J Neuropathol Exp Neurol. 1996. 55(10): 1083-8.

51. Webster, S., et al. Molecular and Cellular Characterization of the Membrane Attack Complex, C5b-9, in Alzheimer's Disease. Neurobiology of Aging. 1997; 18(4): 415-421.

52. Togo, T., et al. Occurrence of T cells in the brain of Alzheimer's disease and other neurological diseases. Journal of Neuroimmunology. 2002;124(1-2): 83-92.

53. Saresella, M. Increased activity of Th-17 and Th-9 lymphocytes and a skewing of the post-thymic differentiation pathway are seen in Alzheimer's disease. Brain, Behavior, and Immunity. 2011 25(3): 539547.

54. Saresella, M. PD1 negative and PD1 positive CD4+ T regulatory cells in mild cognitive impairment and Alzheimer's disease. J Alzheimers Dis. 2010; 21(3): 927-38.
55. Bö, L., et al. Grey matter pathology in multiple sclerosis. Acta Neurologica Scandinavica. 2006; 113: 48-50.

56. McFarland, H.F. and R. Martin. Multiple sclerosis: a complicated picture of autoimmunity. Nat Immunol. 2007; 8(9): 913-9.

57. van Horssen, J., et al. Radical changes in multiple sclerosis pathogenesis. Biochimica et Biophysica Acta (BBA) - Molecular Basis of Disease. 2011; 1812(2): 141-150.

58. Tienari, P.J. Multiple Sclerosis: Multiple Etiologies, Multiple Genes? Annals of Medicine. 1994; 26(4): 259-269.

59. Hafler, D.A. Multiple sclerosis. The Journal of Clinical Investigation. 2004; 113(6): 788-794

60. Noseworthy, J.H. Multiple Sclerosis. New England Journal of Medicine 2000; 343(13): 938-952.

61. Bö, L. Subpial demyelination in the cerebral cortex of multiple sclerosis patients. J Neuropathol Exp Neurol. 2003; 62(7): 723-32.

62. Frohman, E.M., M.K. Racke, and C.S. Raine. Multiple Sclerosis â€" The Plaque and Its Pathogenesis. New England Journal of Medicine. 2006;354(9): 942-955.

63. The International_Multiple_Sclerosis Genetics_Consortium and The_Wellcome_Trust_Case_Control_Consortium_2, Genetic risk and a primary role for cell-mediated immune mechanisms in multiple sclerosis. Nature. 2011; 476(7359): 214-219.

64. Handel, A.E. Smoking and Multiple Sclerosis: An Updated MetaAnalysis. PLOS ONE. 2011; 6(1): 16149.

65. Munger, K.L. Serum 25-Hydroxyvitamin D Levels and Risk of Multiple Sclerosis. JAMA The Journal of the American Medical Association. 2006; 296(23): 2832-2838.

66. Sundström, P. An altered immune response to Epstein-Barr virus in multiple sclerosis. Neurology. 2004; 62(12): 2277-2282.

67. Thacker, E.L., F. Mirzaei, and A. Ascherio. Infectious mononucleosis and risk for multiple sclerosis: A meta-analysis. Annals of Neurology. 2006; 59(3): 499-503.

68. O'Connor, K.C., A. Bar-Or, and D.A. Hafler. The Neuroimmunology of Multiple Sclerosis: Possible Roles of $T$ and B Lymphocytes in Immunopathogenesis. Journal of Clinical Immunology. 2001; 21(2): 81-92.

69. Comabella, M. and S.J. Khoury. Immunopathogenesis of multiple sclerosis. Clinical Immunology. 2011: In Press, Corrected Proof

70. Diaz-Villoslada, P. Autoreactivity to myelin antigens: myelin/ oligodendrocyte glycoprotein is a prevalent autoantigen. Journal of Neuroimmunology. 1999; 99(1): 36-43.

71. Lovett-Racke, A.E. Decreased dependence of myelin basic proteinreactive $T$ cells on $\mathrm{CD} 28$-mediated costimulation in multiple sclerosis patients. A marker of activated/memory T cells. The Journal of Clinical Investigation. 1998; 101(4): 725-730.

72. Babbe, H. Clonal Expansions of Cd8+ T Cells Dominate the T Cell Infiltrate in Active Multiple Sclerosis Lesions as Shown by Micromanipulation and Single Cell Polymerase Chain Reaction. The Journal of Experimental Medicine. 2000; 192(3): 393-404

73. Mosmann, T.R. Two types of murine helper T cell clone. I. Definition according to profiles of lymphokine activities and secreted proteins. The Journal of Immunology. 1986; 136(7): 2348-2357.

74. Panitch, H.S. Treatment of multiple sclerosis with gamma interferon Neurology. 1987; 37(7): 1097.

75. Lassmann, H. and R.M. Ransohoff. The CD4-Th1 model for multiple sclerosis: a crucial re-appraisal. Trends in Immunology. 2004; 25(3): 132-137.

76. Brucklacher-Waldert, V. Phenotypical and functional characterization of T helper 17 cells in multiple sclerosis. Brain. 2009; 132(12): 33293341. 
77. Viglietta, V. Loss of Functional Suppression by CD4+CD25+ Regulatory T Cells in Patients with Multiple Sclerosis. The Journal of Experimental Medicine. 2004; 199(7): 971-979.

78. Neumann, H. Cytotoxic T lymphocytes in autoimmune and degenerative CNS diseases. Trends Neurosci. 2002; 25(6): 313-9.

79. Forno, L.S. Neuropathology of Parkinson's disease. J Neuropathol Exp Neurol. 1996; 55(3): 259-72.

80. Olanow, C.W. and W.G. Tatton. ETIOLOGY AND PATHOGENESIS OF PARKINSON'S DISEASE. Annual Review of Neuroscience. 1999; 22(1): 123-144.

81. Irvine, G.B. Protein aggregation in the brain: the molecular basis for Alzheimer's and Parkinson's diseases. Mol Med. 2008; 14(7-8): 45164.

82. Obeso, J.A. Missing pieces in the Parkinson's disease puzzle. Nat Med. 2010; 16(6): 653-661.

83. Koller, W. Environmental risk factors in Parkinson's disease. Neurology. 1990; 40(8): 1218-21.

84. Tanner, C.M. and J.W. Langston. Do environmental toxins cause Parkinson's disease? A critical review. Neurology. 1990; 40(10 Suppl 3): suppl 17-31.

85. Golbe, L.I. The genetics of Parkinson's disease: a reconsideration. Neurology. 1990; 40(10 Suppl 3): 7-14, 14-6.

86. Hunot, S. and E.C. Hirsch. Neuroinflammatory processes in Parkinson's disease. Annals of Neurology. 2003; 53(S3): S49-S60.

87. Imamura, K. Distribution of major histocompatibility complex class IIpositive microglia and cytokine profile of Parkinson's disease brains. Acta Neuropathologica. 2003; 106(6): 518-526.

88. Lee, J.-K., T. Tran, and M. Tansey. Neuroinflammation in Parkinsonâ€ $€^{\mathrm{TM} S}$ Disease. Journal of Neuroimmune Pharmacology. 2009; 4(4): 419429.

89. McGeer, P.L. Reactive microglia are positive for HLA-DR in the substantia nigra of Parkinson's and Alzheimer's disease brains. Neurology. 1988;38(8): 1285-91.

90. Kurkowska-Jastrzebska, I. The Inflammatory Reaction Following 1-Methyl-4-phenyl-1,2,3,6-tetrahydropyridine Intoxication in Mouse. Experimental Neurology. 1999; 156(1): 50-61.

91. Ransohoff, R.M. and V.H. Perry. Microglial physiology: unique stimuli, specialized responses. Annu Rev Immunol. 2009; 27: 119-45.

92. Le, W.-d., et al. Microglial Activation and Dopaminergic Cell Injury: An In Vitro Model Relevant to Parkinson's Disease. The Journal of Neuroscience. 2001;21(21): 8447-8455.

93. Ciesielska, A. Dynamics of expression of the mRNA for cytokines and inducible nitric synthase in a murine model of the Parkinson's disease. Acta Neurobiol Exp (Wars). 2003; 63(2): 117-26.

94. Griffin, W.S. Interleukin-1 mediates Alzheimer and Lewy body pathologies. Journal of Neuroinflammation. 2006; 3(1): 5

95. Knott, C., G. Stern, and G.P. Wilkin. Inflammatory Regulators in Parkinson's Disease: iNOS, Lipocortin-1, and Cyclooxygenases-1 and -2. Molecular and Cellular Neuroscience. 2000; 16(6): 724-739.

96. McGeer, E.G., A. Klegeris, and P.L. McGeer. Inflammation, the complement system and the diseases of aging. Neurobiology of Aging. 2005; 26(1, Supplement): 94-97.

97. Klegeris, A. [alpha]-Synuclein activates stress signaling protein kinases in THP-1 cells and microglia. Neurobiology of Aging. 2008; 29(5): 739752.

98. McGeer, P.L. and E.G. McGeer. Inflammation and neurodegeneration in Parkinson's disease. Parkinsonism \& Related Disorders. 2004; 10(Supplement 1): p. S3-S7.

99. Liberatore, G.T. Inducible nitric oxide synthase stimulates dopaminergic neurodegeneration in the MPTP model of Parkinson disease. Nat Med. 1999; 5(12): 1403-1409.
100. Engelhardt, B. Immune cell entry into the central nervous system: Involvement of adhesion molecules and chemokines. Journal of the Neurological Sciences. 2008; 274(1-2): 23-26.

101. Brochard, V. Infiltration of CD4+ lymphocytes into the brain contributes to neurodegeneration in a mouse model of Parkinson disease. J Clin Invest. 2009; 119(1): 182-92.

102. Chung, Y.C. The role of neuroinflammation on the pathogenesis of Parkinson's disease. BMB Rep. 2010; 43(4): 225-32.

103. Huang, X. CD 4+T cells in the pathobiology of neurodegenerative disorders. Journal of Neuroimmunology. 2009; 211(1-2): 3-15.

104. Rezai-Zadeh, K., D. Gate, and T. Town. CNS Infiltration of Peripheral Immune Cells: D-Day for Neurodegenerative Disease? Journal of Neuroimmune Pharmacology. 2009; 4(4): 462-475.

105. Reynolds, A.D. Regulatory T Cells Attenuate Th17 CellMediated Nigrostriatal Dopaminergic Neurodegeneration in a Model of Parkinsonâ $€^{\mathrm{TM}} \mathrm{s}$ Disease. The Journal of Immunology. 2010; 184(5): 2261-2271.

106. Reynolds, A.D. Nitrated alpha-Synuclein-Induced Alterations in Microglial Immunity Are Regulated by CD4+ T Cell Subsets. The Journal of Immunology. 2009. 182(7): 4137-4149.

107. Reynolds, A.D. Neuroprotective activities of CD4+CD25+ regulatory T cells in an animal model of ParkinsonÂ's disease. Journal of Leukocyte Biology. 2007; 82(5):1083-1094.

108. Warlow, C. Stroke. The Lancet. 2003; 362(9391): 1211-1224.

109. Donnan, G.A. Stroke. The Lancet. 2008; 371(9624): 1612-1623.

110. Auer, R.N. and G.R. Sutherland. Primary intracerebral hemorrhage: pathophysiology. Can J Neurol Sci. 2005; 32 Suppl 2: S3-12.

111. Flynn, R.W.V., R.S.M. MacWalter, and A.S.F. Doney. The cost of cerebral ischaemia. Neuropharmacology. 2008; 55(3): 250-256.

112. Hossmann, K.A.. Viability thresholds and the penumbra of focal ischemia. Annals of Neurology. 1994; 36(4): 557-565.

113. Brouns, R. and P.P. De Deyn. The complexity of neurobiological processes in acute ischemic stroke. Clin Neurol Neurosurg. 2009; 111(6): 483-95.

114. Sims, N.R. and H. Muyderman. Mitochondria, oxidative metabolism and cell death in stroke. Biochimica et Biophysica Acta (BBA) - Molecular Basis of Disease. 2010; 1802(1): 80-91.

115. Denes, A. Inflammation and brain injury: Acute cerebral ischaemia, peripheral and central inflammation. Brain, Behavior, and Immunity. 2010; 24(5): 708-723.

116. McColl, B.W., S.M. Allan, and N.J. Rothwell. Systemic inflammation and stroke: aetiology, pathology and targets for therapy. Biochem Soc Trans. 2007; 35(Pt 5): 1163-5.

117. McColl, B.W., S.M. Allan, and N.J. Rothwell. Systemic infection, inflammation and acute ischemic stroke. Neuroscience. 2009. 158(3): p. 1049-1061.

118. Wang, Q., X.N. Tang, and M.A. Yenari, The inflammatory response in stroke. J Neuroimmunol. 2007; 184(1-2): 53-68.

119. Katsura, K., T. Kristian, and B.K. Siesjo. Energy metabolism, ion homeostasis, and cell damage in the brain. Biochem Soc Trans. 1994; 22(4): 991-6

120. Martin, R.L., H.G.E. Lloyd, and A.I. Cowan. The early events of oxygen and glucose deprivation: setting the scene for neuronal death? Trends in neurosciences. 1994; 17(6): 251-257.

121. Nicholls, D. and D. Attwell. The release and uptake of excitatory amino acids. Trends in Pharmacological Sciences. 1990; 11(11): 462468.

122. Choi, D.W. Calcium-mediated neurotoxicity: relationship to specific channel types and role in ischemic damage. Trends in neurosciences. 1988; 11(10): 465-469. 
123. Meldrum, B. ISCHAEMIC BRAIN DAMAGE: THE ROLE OF EXCITATORY ACTIVITY AND OF CALCIUM ENTRY. British Journal of Anaesthesia. 1985; 57(1): 44-46.

124. Rothman, S.M. and J.W. Olney. Glutamate and the pathophysiology of hypoxic-ischemic brain damage. Annals of Neurology. 1986. 19(2): 105-111.

125. Coyle, J.T. and P. Puttfarcken. Oxidative stress, glutamate, and neurodegenerative disorders. Science. 1993; 262(5134):689-95.

126. Langley, B. and R.R. Ratan. Oxidative stress-induced death in the nervous system: Cell cycle dependent or independent? Journal of Neuroscience Research. 2004; 77(5): 621-629.

127. Chan, P.H. Reactive Oxygen Radicals in Signaling and Damage in the Ischemic Brain. J Cereb Blood Flow Metab. 2001; 21(1): 2-14.

128. Love, S. Oxidative Stress in Brain Ischemia. Brain Pathology. 1999; 9(1): p. 119-131.

129. Nelson, C.W. Oxygen radicals in cerebral ischemia. American Journal of Physiology - Heart and Circulatory Physiology. 1992; 263(5): H1356-H1362.

130. Dong, Y. and E.N. Benveniste. Immune function of astrocytes. Glia. 2001; 36(2): 180-190

131. Morioka, T., A.N. Kalehua, and W.J. Streit. Characterization of microglial reaction after middle cerebral artery occlusion in rat brain. The Journal of Comparative Neurology. 1993; 327(1): 123-132.

132. Liu, T. Interleukin-1 beta mRNA expression in ischemic rat cortex. Stroke. 1993; 24(11): 1746-1750.

133. Minami, M. Induction of Interleukin-1B mRNA in Rat Brain After Transient Forebrain Ischemia. Journal of Neurochemistry. 1992; 58(1): 390-392

134. Brough, D., P.J. Tyrrell, and S.M. Allan. Regulation of interleukin-1 in acute brain injury. Trends in Pharmacological Sciences. 2011; 32(10): 617-622.

135. Yan, J. Immune activation in the peripheral blood of patients with acute ischemic stroke. Journal of Neuroimmunology. 2009; 206(1-2):112-117.

136. Offner, H. Splenic Atrophy in Experimental Stroke Is Accompanied by Increased Regulatory $T$ Cells and Circulating Macrophages. The Journal of Immunology. 2006. 176(11): 6523-6531.

137. Blann, A.D., P.M. Ridker, and G.Y.H. Lip. Inflammation, Cell Adhesion Molecules, and Stroke: Tools in Pathophysiology and Epidemiology? Stroke. 2002; 33(9): 2141-2143.

138. Engelhardt, B., F.K. Conley, and E.C. Butcher. Cell adhesion molecules on vessels during inflammation in the mouse central nervous system. Journal of Neuroimmunology. 1994; 51(2): 199-208.

139. Stoll, G., S. Jander, and M. Schroeter. Inflammation and glial responses in ischemic brain lesions. Prog Neurobiol. 1998; 56(2): 14971.

140. Tanne, D. Soluble Intercellular Adhesion Molecule-1 and Risk of Future Ischemic Stroke. Stroke. 2002; 33(9): 2182-2186.

141. Fumagalli, S. In Vivo Real-Time Multiphoton Imaging of $T$ Lymphocytes in the Mouse Brain After Experimental Stroke. Stroke. 2011; 42(5): 1429-1436.

142. Gee, J.M. Lymphocytes: Potential Mediators of Postischemic Injury and Neuroprotection. Stroke, 2007. 38(2): p. 783-788.

143. Ortolano, F. Advances in imaging of new targets for pharmacological intervention in stroke: real-time tracking of T-cells in the ischaemic brain. British Journal of Pharmacology. 2010; 159(4): 808-811.

144. Schroeter, M. Local immune responses in the rat cerebral cortex after middle cerebral artery occlusion. Journal of Neuroimmunology. 1994; 55(2): 195-203
145. Hurn, P.D. T- and B-cell-deficient mice with experimental stroke have reduced lesion size and inflammation. J Cereb Blood Flow Metab. 2007; 27(11): 1798-1805

146. Liesz, A. Inhibition of lymphocyte trafficking shields the brain against deleterious neuroinflammation after stroke. Brain. 2011; 134(3): 704-720.

147. Frenkel, D. Neuroprotection by IL-10-producing MOG CD4+ T cells following ischemic stroke. J Neurol Sci. 2005; 233(1-2): 125-32.

148. Planas, A.M. and A. Chamorro. Regulatory T cells protect the brain after stroke. Nat Med. 2009; 15(2): 138-139.

149. Ren, X. Regulatory B Cells Limit CNS Inflammation and Neurologic Deficits in Murine Experimental Stroke. The Journal of Neuroscience. 2011; 31(23): 8556-8563.

150. Engelhardt, B., Molecular mechanisms involved in $\mathrm{T}$ cell migration across the blood-brain barrier. J Neural Transm. 2006; 113(4): 477-85.

151. Engelhardt, B. and R.M. Ransohoff. The ins and outs of T-lymphocyte trafficking to the CNS: anatomical sites and molecular mechanisms. Trends Immunol. 2005; 26(9): 485-95.

152. Fee, D. Activated/effector CD4+ T cells exacerbate acute damage in the central nervous system following traumatic injury. $J$ Neuroimmunol. 2003; 136(1-2): 54-66.

153. Appel, S.H. CD4+ $T$ cells mediate cytotoxicity in neurodegenerative diseases. J Clin Invest. 2009; 119(1): 13-5.

154. Popovich, P.G., P. Wei, and B.T. Stokes. Cellular inflammatory response after spinal cord injury in Sprague-Dawley and Lewis rats. J Comp Neurol. 1997; 377(3): 443-64.

155. Popovich, P.G., B.T. Stokes, and C.C. Whitacre. Concept of autoimmunity following spinal cord injury: Possible roles for $\mathrm{T}$ lymphocytes in the traumatized central nervous system. Journal of Neuroscience Research. 1996; 45(4): 349-363.

156. Wang, W.Z. Myelin antigen reactive T cells in cerebrovascular diseases. Clinical \& Experimental Immunology. 1992; 88(1): 157-162.

157. Olsson, T. Autoreactive $\mathrm{T}$ and $\mathrm{B}$ cell responses to myelin antigens after diagnostic sural nerve biopsy. Journal of the Neurological Sciences. 1993; 117(1-2): 130-139.

158. Kil, K. T cell responses to myelin basic protein in patients with spinal cord injury and multiple sclerosis. Journal of Neuroimmunology. 1999; 98(2): 201-207.

159. Viviani, B. Cytokines role in neurodegenerative events. Toxicol Lett. 2004; 149(1-3): 85-9.

160. Hsu, H. TRADD TRAF2 and TRADD FADD Interactions Define Two Distinct TNF Receptor 1 Signal Transduction Pathways. Cell. 1996. 84(2): 299-308.

161. Mogi, M. Caspase activities and tumor necrosis factor receptor R1 (p55) level are elevated in the substantia nigra from Parkinsonian brain. Journal of Neural Transmission. 2000; 107(3): 335-341.

162. Dittel, B.N. Evidence that Fas and FasL contribute to the pathogenesis of experimental autoimmune encephalomyelitis. Arch Immunol Ther Exp (Warsz). 2000. 48(5): 381-8.

163. Nagata, S. and T. Suda, Fas and Fas ligand: Ipr and gld mutations. Immunol Today. 1995; 16(1): 39-43.

164. Nagata, S. and P. Golstein. The Fas death factor. Science. 1995; 267(5203): 1449-1456

165. Sabelko-Downes, K.A., A.H. Cross, and J.H. Russell. Dual role for Fas ligand in the initiation of and recovery from experimental allergic encephalomyelitis. J Exp Med. 1999; 189(8): 1195-205.

166. Medana, I. Transection of major histocompatibility complex class I-induced neurites by cytotoxic T lymphocytes. Am J Pathol. 2001; 159(3): 809-15. 
167. Medana, I.M. MHC class I-restricted killing of neurons by virusspecific CD8+ T lymphocytes is effected through the Fas/FasL, but not the perforin pathway. European Journal of Immunology. 2000; 30(12): 3623-3633.

168. Neumann, H. Induction of MHC class I genes in neurons. Science. 1995; 269(5223): 549-552.

169. Rensing-Ehl, A. Neurons induced to express major histocompatibility complex class I antigen are killed via the perforin and not the Fas (APO-1/CD95) pathway. European Journal of Immunology. 1996; 26(9): 2271-2274.

170. Giuliani, F. Vulnerability of Human Neurons to T Cell-Mediated Cytotoxicity. The Journal of Immunology. 2003; 171(1): 368-379.

171. Beers, D.R. CD4+ T cells support glial neuroprotection, slow disease progression, and modify glial morphology in an animal model of inherited ALS. Proceedings of the National Academy of Sciences. 2008; 105(40): 15558-15563.

172. Moalem, G. Production of neurotrophins by activated T cells: implications for neuroprotective autoimmunity. J Autoimmun. 2000; 15(3): 331-45.

173. Moalem, G. Autoimmune $\mathrm{T}$ cells protect neurons from secondary degeneration after central nervous system axotomy. Nat Med. 1999; 5(1): 49-55.

174. Schwartz, M. T-cell-based vaccination against neurodegeneration: a new therapeutic approach. Retina. 2005; 25(8 Suppl):S33-S35.

175. Wolf, S.A. Neuroprotection by T-cells depends on their subtype and activation state. J Neuroimmunol. 2002; 133(1-2): 72-80.

176. Serpe, C.J. CD4+ T, but not CD8+ or B, lymphocytes mediate facial motoneuron survival after facial nerve transection. Brain, Behavior, and Immunity. 2003; 17(5):393-402.

177. Serpe, C.J. Exacerbation of facial motoneuron loss after facial nerve transection in severe combined immunodeficient (scid) mice. $J$ Neurosci. 1999; 19(11): RC7.

178. Hauben, E. Passive or active immunization with myelin basic protein promotes recovery from spinal cord contusion. I Neurosci. 2000; 20(17): 6421-30.

179. Hauben, E. Autoimmune T cells as potential neuroprotective therapy for spinal cord injury. Lancet. 2000; 355(9200): 286-7.

180. Gimsa, U. Th2 cells support intrinsic anti-inflammatory properties of the brain. Journal of Neuroimmunology. 2001; 119(1): 73-80.

181. Bettelli, E. Reciprocal developmental pathways for the generation of pathogenic effector $\mathrm{TH} 17$ and regulatory T cells. Nature. 2006; 441(7090): 235-8.

182. Chen, W. Conversion of Peripheral CD4+CD25â^^ Naive T Cells to CD4+CD25+ Regulatory T Cells by TGF- $\left.\right|^{2}$ Induction of Transcription Factor Foxp3. The Journal of Experimental Medicine. 2003; 198(12): 1875-1886.

183. Nakamura, K., A. Kitani, and W. Strober. Cell Contact-Dependent Immunosuppression by $\mathrm{Cd} 4+\mathrm{Cd} 25+$ Regulatory T Cells Is Mediated by Cell Surface-Bound Transforming Growth Factor-beta. The Journal of Experimental Medicine. 2001; 194(5): 629-644.
184. Liu, Y. Neuron-mediated generation of regulatory T cells from encephalitogenic T cells suppresses EAE. Nat Med. 2006; 12(5):51825.

185. Liu, J. Neuromodulatory Activities of CD4+CD25+ Regulatory T Cells in a Murine Model of HIV-1-Associated Neurodegeneration. J Immunol. 2009; 182(6): 3855-3865.

186. Weiner, H.L. Induction and mechanism of action of transforming growth factor- $\beta$-secreting Th3 regulatory cells. Immunological Reviews 2001; 182(1): 207-214.

187. Xin, J. IL-10 within the CNS is necessary for CD4+ T cells to mediate neuroprotection. Brain, Behavior, and Immunity. 2011; 25(5): 820-829.

188. Hammarberg, H. Neuroprotection by Encephalomyelitis: Rescue of Mechanically Injured Neurons and Neurotrophin Production by CNS-Infiltrating T and Natural Killer Cells. The Journal of Neuroscience. 2000; 20(14): 5283-5291.

189. Shaked, I. Protective autoimmunity: interferon-gamma enables microglia to remove glutamate without evoking inflammatory mediators. J Neurochem. 2005; 92(5): 997-1009.

190. Hjelmström, P. Cutting Edge: B Cell-Deficient Mice Develop Experimental Allergic Encephalomyelitis with Demyelination After Myelin Oligodendrocyte Glycoprotein Sensitization. The Journal of Immunology. 1998; 161(9): 4480-4483.

191. Wolf, S.D. Experimental autoimmune encephalomyelitis induction in genetically B cell-deficient mice. J Exp Med. 1996; 184(6): 2271-8.

192. Matsushita, T. Inhibitory role of CD19 in the progression of experimental autoimmune encephalomyelitis by regulating cytokine response. Am J Pathol. 2006; 168(3): 812-21.

193. Matsushita, T. Regulatory $B$ cells inhibit EAE initiation in mice while other B cells promote disease progression. J Clin Invest. 2008; 118(10): 3420-30.

194. Fillatreau, S. B cells regulate autoimmunity by provision of IL10. Nat Immunol. 2002; 3(10): 944-950.

195. Lyons, J.-A. B cells limit epitope spreading and reduce severity of EAE induced with PLP peptide in BALB/c mice. Journal of Autoimmunity. 2008; 31(2): 149-155.

196. Edling, A.E. Human and murine lymphocyte neurotrophin expression is confined to B cells. J Neurosci Res. 2004; 77(5): 709-17.

197. Kala, M. B cells from glatiramer acetate-treated mice suppress experimental autoimmune encephalomyelitis. Exp Neurol. 2010; 221(1): 136-45.

198. Kerschensteiner M. Activated human T cells, B cells, and monocytes produce brain-derived neurotrophic factor in vitro and in inflammatory brain lesions: a neuroprotective role of inflammation? J Exp Med. 1999; 189(5): 865-70.

199. Shrestha R, O. Millington J, Brewer ,K K. Dev and T. J. Bushell (2014). "Lymphocyte-mediated neuroprotection in in vitro models of excitotoxicity involves astrocytic activation and the inhibition of MAP kinase signalling pathways." Neuropharmacology. 76 Pt A: 184-193. 